\title{
Epitaxial Germanium Nanowires on GaAs Grown by Chemical Vapor Deposition
}

\author{
Yong KIm,* Man Suk Song, Young Dae KIM and Jae Hun Jung \\ Department of Physics, Dong-A University, Busan 604-714 \\ Q. GaO, H. H. TAN and C. Jagadish \\ Department of Electronic Materials Engineering, Research School of Physical Sciences and Engineering, \\ The Australian National University, Canberra, ACT0200, Australia
}

(Received 7 March 2007)

\begin{abstract}
We successfully synthesized epitaxial Ge nanowires on GaAs (100) and GaAs (111)B substrates by using Au-nanoparticle-catalyzed chemical vapor deposition. From an analysis of the inclined angles of the Ge nanowires to the substrate normal, we find that the epitaxial Ge nanowires grow along the $\langle 110\rangle$ directions regardless of the substrate's orientation. This is in contrast with epitaxial Ge nanowires on $\mathrm{Si}$ and Ge substrates, which grow predominantly along the $<111>$ direction. In addition, tapering of the Ge nanowires is minimal even at relatively high growth temperatures due to the low surface mobility of the Ge adatoms on the GaAs surface.
\end{abstract}

PACS numbers: 61.46.-w, 72.80.Cw, 81.15.Gh

Keywords: Nanowire, Germanium, GaAs, Chemical vapor deposition

\section{INTRODUCTION}

Si has played a major role in today's microelectronics even though the first transistor was made by Ge. This stems from the fact that the growth of a dense and reliable oxide suitable for an insulating layer in a metal-oxide semiconductor field-effect transistor (MOSFET) is relatively straightforward by using dry and wet oxidation processes [1]. Ge has regained interest recently as active channel material in nanoscale complementary MOSFET because of progressive development of high dielectric constant materials. Ge is an ideal candidate for high-speed switching devices due to its superior intrinsic carrier mobility compared to that of silicon [2]. Besides the microelectronics applications, Ge is also attractive for optoelectronic applications. For instance, due to its strong absorption in the spectral range of $1.0-1.5 \mu \mathrm{m}$ and high refractive index $(n=4.0)$, Ge is a promising material for mid-infrared detection and effective wave guides. In addition, the excitonic Bohr radius is significantly larger for Ge $(24.3 \mathrm{~nm})$ as compared to $\mathrm{Si}$ $(4.7 \mathrm{~nm})$; thus, the quantum confinement effect is easily achievable. The lattice mismatch between Ge and GaAs is virtually absent $(\sim 0.1 \%)$. As a natural consequence, there have been several attempts to realize high-quality Ge layers on GaAs substrates for the realization of monolithically integrated optoelectronic devices. However, the

*E-mail: yongkim@dau.ac.kr; Fax: +82-51-200-7232 incorporation of a high concentration of $\mathrm{As} / \mathrm{Ga}$ in a $\mathrm{Ge}$ layer due to outdiffusion from the GaAs substrate has been recognized as a major issue [3]. The incorporation of As/Ga produces a high level of strain in the layer and leads to poor surface morphology, which limits the application of Ge layers to devices. One way to suppress the outdiffusion is to reduce the growth temperature to less than $400{ }^{\circ} \mathrm{C}$. However, a low growth temperature usually leads to poor crystalline quality [4].

The anisotropic growth of silicon whiskers via the vapor-liquid-sold (VLS) growth mechanism was discovered by Wagner and Ellis almost four decades ago [5] According to this mechanism, a small Au particle on a semiconductor surface forms a eutectic liquid alloy with the host material at the growth temperature. The liquid droplet is readily supersaturated with reaction species supplied from the surrounding vapor. The precipitation of supersaturated species at the solid-liquid interface leads to highly anisotropic whisker growth. Nanowires, scaled down in dimensions from these micrometer-sized whiskers, have gained much interest due to their potential as nano-building blocks [6]. Nanowire devices, such as nanowire lasers $[7,8]$, resonant tunneling diodes [9], single electron tunneling diodes [10], and nanowire avalanche photodiodes [11], have been demonstrated. Nanowires have been synthesized by using various methods, including laser ablation [12], molecular beam epitaxy [13], thermal evaporation [14,15], and metalorganic chemical vapor deposition (MOCVD) $[16,17]$. 
Non-epitaxial Ge nanowires, which are not connected atomically with the underlying substrate, are usually entangled, despite the perfect crystalline property and uniform diameter [18]. Therefore, epitaxial Ge nanowires are more desirable for on-chip integration of nanowirebased optoelectronic devices. Epitaxial Ge nanowires grown on $\mathrm{Si}$ and Ge substrates were reported in Refs. 19-24. The preferred growth direction of these epitaxial Ge nanowires was along the $<111>$ direction. The growth temperatures for the Ge nanowires were in the range from $290{ }^{\circ} \mathrm{C} \sim 400{ }^{\circ} \mathrm{C}$. Careful surface preparation to avoid the formation of a native oxide was a crucial step toward the successful growth of an epitaxial Ge nanowire specifically on a Si substrate [23].

As/Ga incorporation in a Ge layer on GaAs during the growth at high temperatures $\left(>400{ }^{\circ} \mathrm{C}\right)$ is a major obstacle for achieving a high-quality Ge layer. The Ge nanowire growth temperatures reported in the literature were at least $100{ }^{\circ} \mathrm{C}$ lower than the conventional Ge layer growth temperatures. Therefore, minimal As/Ga ncorporation in the Ge nanowire is expected. Furthermore, negligible As desorption from the GaAs surface at these low growth temperatures may ensure a good surface morphology in the region without nanowires. In addition, the oxidation on the GaAs surface during preparation process is slow. The slow oxidation rate could result in a high production yield. These ideas along with negligible lattice mismatch between Ge and GaAs motivated the present study of epitaxial Ge nanowires on GaAs substrates.

\section{EXPERIMENTS}

Undoped GaAs (100) and (111)B substrates were functionalized by dipping them in a $0.1 \%$ poly-L-lysine (PLL) solution for 1 minute. After having been rinsed with deionized (DI) water, the substrates were blown dry with $\mathrm{N}_{2}$ gas. A commercially available $\mathrm{Au}$ colloidal liquid solution (Ted Pellar, Inc.) containing $50 \mathrm{~nm} \pm 3 \mathrm{~nm}$ diameter Au nanoparticles with the density of $4.5 \times 10^{10}$ particles $/ \mathrm{ml}$ was dispersed on the substrate surface and rinsed off with DI water after $30 \mathrm{~s}$. The few-monolayerthick PLL layer acts as a positively charged polyelectrolyte and attracts negatively charged $\mathrm{Au}$ nanoparticles and immobilizes them on the substrate surface. The prepared substrates were immediately loaded in a coldwall stainless-steel reaction chamber. Ge nanowires were grown by using a lamp-heated chemical vapor deposition system. The processing gas was $1 \% \mathrm{H}_{2}$-diluted $\mathrm{GeH}_{4}$. A bank of tungsten-halogen lamps allowed rapid heating of the substrates at a ramp rate of $80^{\circ} \mathrm{C} / \mathrm{min}$. Wafer temperatures, calibrated by using a thermocouple, were monitored by an infrared pyrometer (IRCON with a $5 \mu \mathrm{m}$ detection band) through a sapphire window. The chamber pressure was maintained by using the feedback from a throttle valve (MKS) and a capac- itance manometer (Baratron). The chamber pressure was kept at 10 Torr $\left(\mathrm{GeH}_{4}\right.$ partial pressure $=0.1$ Torr $)$ throughout the experiment. The growth temperatures were in the range from $300{ }^{\circ} \mathrm{C} \sim 380{ }^{\circ} \mathrm{C}$, and the temperatures were changed in steps of $10{ }^{\circ} \mathrm{C}$ or $15{ }^{\circ} \mathrm{C}$. A growth time of 1 hour was used. For comparison purposes, Ge nanowire growth on a Si substrate was also tried. Si (100) substrates were etched by using HF to remove the native oxide after the conventional RCA cleaning step. The Si surface was functionalized by dipping it in aminopropyltriethoxysilane for 1 minute. Some of the $\mathrm{Au}$-nanoparticle-dispersed samples were further furnaceannealed in a $\mathrm{H}_{2}$ ambient for $40 \mathrm{~min}$. at $550{ }^{\circ} \mathrm{C}$. The growth conditions were equivalent to those for $\mathrm{GaAs}$ substrates, except for the growth time (2 hours). The top-view and cross-sectional views of the samples were observed by using a field emission scanning electron microscope (FESEM) (JEOL, JSM-6700F).

\section{RESULTS AND DISCUSSION}

An extensive study of epitaxial Ge nanowire growth on a Si substrate was carried out initially. HF etching is known to provide a hydrogen-passivated surface, which keeps the surface from further oxidation for a few tens of a second. The agglomeration approach after thin $\mathrm{Au}$ film deposition to form $\mathrm{Au}$ nanoparticles on surface is useful to avoid native oxide formation because the sample is immediately loaded into $\mathrm{Au}$ evaporation chamber after native oxide removal [23]. However, the colloid dispersion technique requires, at least, a few minutes for preparation. During this prolonged process, native oxide seems to be formed even though the native oxide had been thoroughly removed by HF etching. Therefore, the production yield of Ge nanowires was very low. We found that furnace annealing in a $\mathrm{H}_{2}$ ambient after $\mathrm{Au}$ colloid dispersion increased the Ge nanowire production yield drastically.

Figure 1 shows the top view and the cross-sectional view FESEM images of Ge nanowires on a Si (100) substrate. A high density of Ge nanowires is formed on the surface. However, the distribution of Ge nanowires, as noted in the top view image (Figure 1(a)), is nonuniform, reflecting the $\mathrm{Au}$ agglomeration process during the annealing. The increase in the production yield may be attributed to partial removal of the native oxide during $\mathrm{H}_{2}$ annealing. Despite the increase in the production yield, the Ge nanowires are still non-epitaxial, as shown in Figure 1(b). Further increasing the annealing temperature only results in significant agglomeration, and no epitaxial Ge nanowires were observed. These are the problems with which we were confronted during the growth of Ge nanowires on Si substrates. However, the result was remarkably different when we tried to grow Ge nanowires on GaAs substrates. This paper demonstrates epitaxial Ge nanowire growth at a growth temperatures 

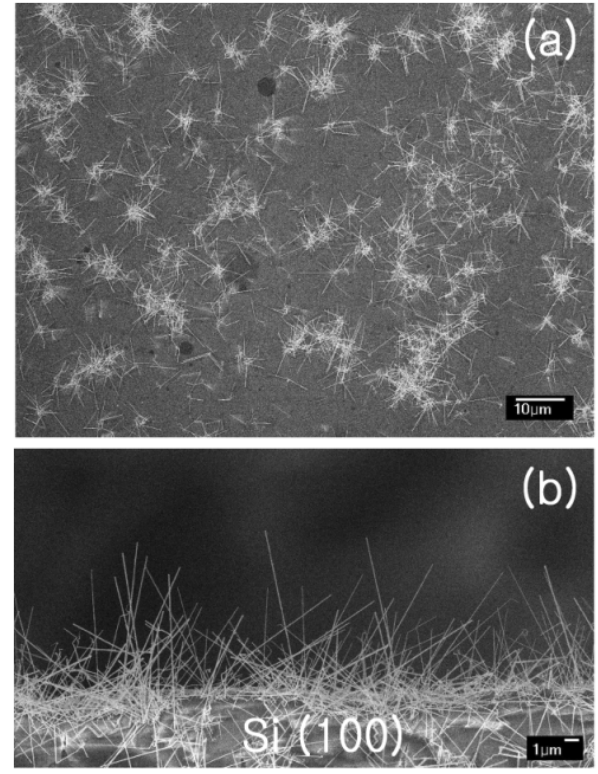

Fig. 1. (a) Top view and (b) cross-sectional view FESEM images of Ge nanowires grown on Si (100) substrates at 350 ${ }^{\circ} \mathrm{C}$.

of $350{ }^{\circ} \mathrm{C}$, which was the optimum temperature in our study. First, the result of Ge nanowires grown on GaAs (100) substrates, which are most widely used, are shown.

Figure 2 shows the top view and the cross-sectional view of Ge nanowires on GaAs (100) grown at $350{ }^{\circ} \mathrm{C}$. Remarkably, all nanowires are epitaxial, judging from the symmetrical arrangement of nanowires in Figure 2(a). The top view images in Figure 2(a) were carefully aligned so that each side of the images was parallel to cleavage direction of the (100) substrate. If Ge nanowires grow along $<111>$ direction, nanowires will arrange parallel to the cleavage direction. However, as noted in Figure 2(a), most of nanowire directions had rectangular symmetry, but were rotated $45^{\circ}$ from the cleavage direction. Referring to Figure 2(c), this configuration is possible when Ge nanowires choose their growth directions along the $\langle 110\rangle$ direction. This result is completely different from those of previous reports. To confirm further, we observed the cross-section view image in Figure 2 (b). The viewing direction is [01-1]. Obvious epitaxial growth of Ge nanowires is confirmed again. The apparent viewing angle of Ge nanowires grown along the [110] direction, considering the $45^{\circ}$ projection of the nanowire image on the plane perpendicular to the viewing direction, can be calculated to be $35.3^{\circ}$. Figure 2(b) shows the cross-sectional view FESEM image of a Ge nanowire grown on $\operatorname{GaAs}(100)$ at $350{ }^{\circ} \mathrm{C}$. The viewing angle between the [111] direction and the Ge nanowire is $36^{\circ}$ and exactly matches (within experimental error) the calculated angle. Therefore, we conclude that Ge nanowires grow along the $\langle 110\rangle$ directions.

In the top view image in Figure 2(a), a small number of nanowires has rectangular symmetry parallel to
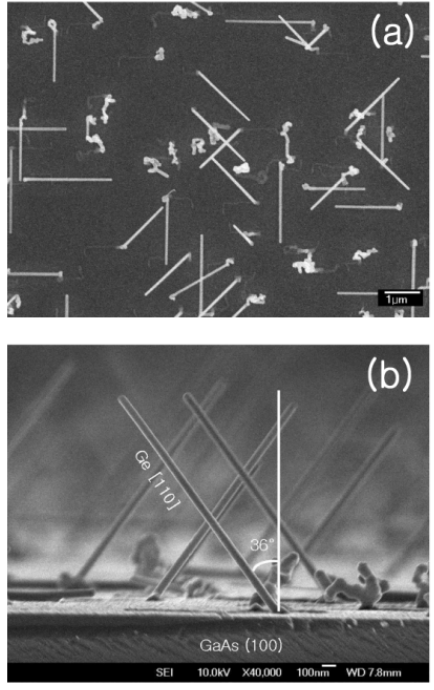

(b)

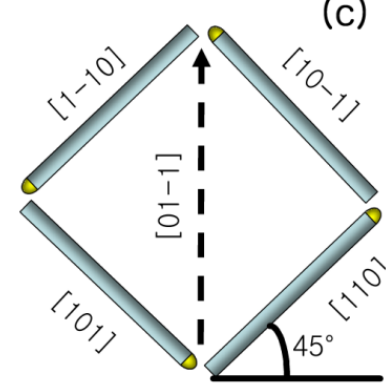

Fig. 2. (a) Top view and (b) cross-sectional view FESEM images of Ge nanowires grown on GaAs (100) substrates at $350{ }^{\circ} \mathrm{C}$. (c) Projected nanowire arrangements on the (100) surface for Ge nanowires grown along the $<110>$ direction. Scale bars are (a) $1 \mu \mathrm{m}$ and (b) $100 \mathrm{~nm}$.

the cleavage direction. Since $<111>$ nanowires will have the same symmetry when projected on (100) surface, we carefully inspected the cross-sectional view of the FESEM images to find $<111>$ nanowires, which are inclined at $54.7^{\circ}$ to the surface normal. However, we did not find such nanowires. Therefore, these nanowires are not $<111>$ nanowires. Tapering is not so significant for our Ge nanowires (59 nm near the tip and $62 \mathrm{~nm}$ near the base) compared to other reports on nanowires grown at similar temperatures $[19,22,23]$. Tapering arises from the radial growth on nanowire side wall of reaction species, which initially arrive on the semiconductor surface and travel toward the nanowires [25]. Therefore, tapering is closely related to the surface mobility of adatoms on semiconductors. The mobility of the Ge adatom on GaAs may be insignificant compared to that on a $\mathrm{Si}$ or a $\mathrm{Ge}$ surface though we did not find an adequate reference. Certainly, tapering is an undesirable property because it leads to nanowires with non-uniform diameters. To avoid this effect, Greytak et al. proposed a two-temperature growth technique, which separated the nucleation and the growth temperatures [26]. The usefulness of this technique is further confirmed by Adhikari et al. [22]. However, this approach is rather complex. Therefore, growing Ge nanowires on GaAs is advantageous in this sense.

The previous Ge nanowire growth on Si and Ge substrates predominantly grew along the $<111>$ directions; thus, the Ge nanowires on (111) substrates were vertically standing. Figure 3(a) shows the top view FESEM image of Ge nanowires on GaAs (111)B substrate grown at $350^{\circ} \mathrm{C}$. If $\mathrm{Ge}$ nanowires grow vertically, nanowires will appear as distinct spots in the top view FESEM images. 

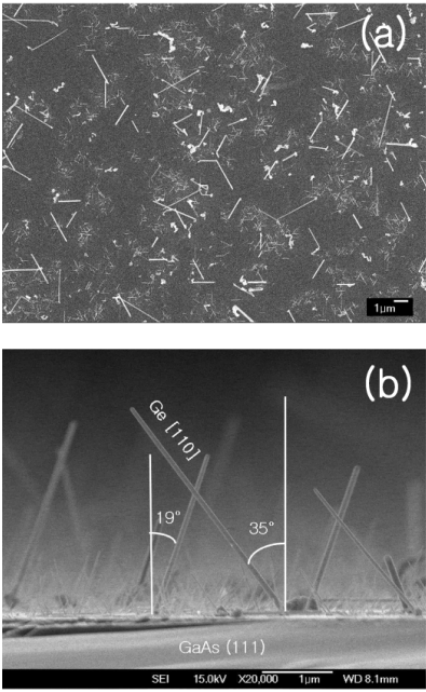
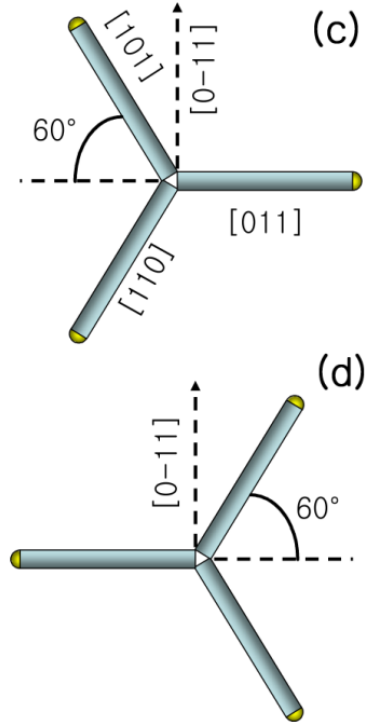

Fig. 3. (a) Top view and (b) cross-sectional view FESEM images of Ge nanowires grown on GaAs (111)B substrates grown at $350{ }^{\circ} \mathrm{C}$. (c) Projected nanowire arrangements on the (111) surface for Ge nanowires grown along the $<110>$ direction. (d) Equivalent crystallographic arrangement of (c). Scale bars are $1 \mu \mathrm{m}$.

We carefully inspect all FESEM images, but there are no such vertical nanowires. All Ge nanowires show triangular symmetry, implying epitaxial growth similar to Ge nanowires on GaAs (100). Interestingly, a group of Ge nanowires with a diameter substantially smaller than the diameter of Au nanoparticles is observed, which seems to have a heterogeneous origin but still have an epitaxial relationship to the underlying substrate. If Ge nanowires on GaAs (111)B grow along the $<110>$ direction as is the case of Ge nanowires on GaAs (100), possible configurations/directions of the Ge nanowires are shown in Figures 3(c) and (d). The configuration of Figure 3(d) is crystallographically equivalent to that of Figure 3(c). Choosing a viewing direction of [0-11], the cross-sectional view FESEM image will show two different nanowire angles, considering the $60^{\circ}$ projection of nanowire images to the plane normal relative to the viewing direction. The calculated angles are $35.3^{\circ}$ and $19.5^{\circ}$, respectively. Figure 3 (b) shows a cross-sectional view FESEM image of Ge nanowires grown at $350{ }^{\circ} \mathrm{C}$. The image clearly confirms the epitaxial growth of Ge nanowires on a GaAs (111)B substrate. The measured viewing angles are $19^{\circ}$ and $35^{\circ}$, which exactly match the expected viewing angles of [110] Ge nanowires. Again, no significant tapering is observed (65 nm diameter near the tip and $85 \mathrm{~nm}$ near the base). In the top view images of Figure 3(a), some Ge nanowires show triangular symmetry, but symmetry triangle is rotated at $30^{\circ}$ as shown in Figures 4 (a) and (b). The nanowires grown along the [111] directions will have the same projected symmetry on the (111) surface. In this case, the viewing angle from the cross-sectional
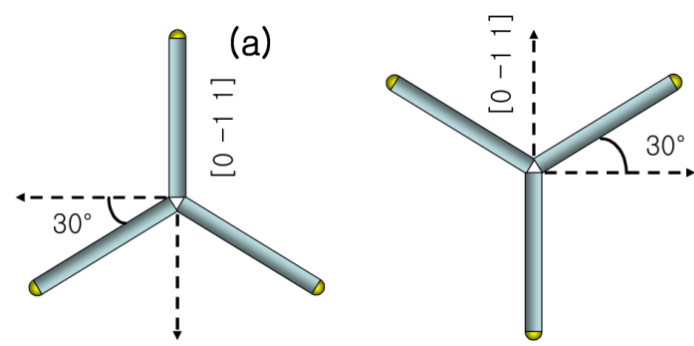

(b)

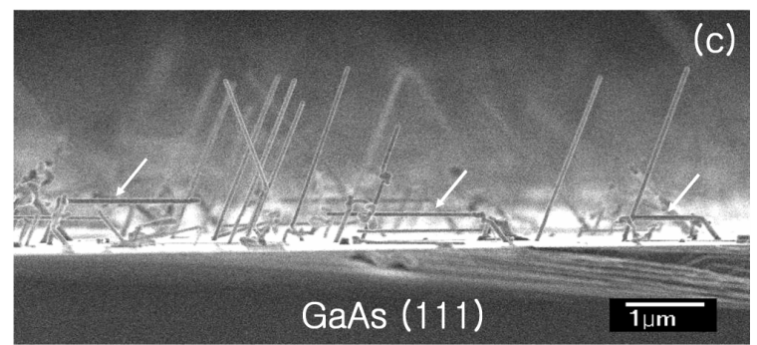

Fig. 4. (a) Projected arrangement on the (111) surface for Ge nanowires grown along the [0-11], [01-1], [1-10], and [-101] directions perpendicular to the surface normal. (b) Equivalent crystallographic arrangement of (a). (c) Crosssectional view FESEM image of Ge nanowires grown on GaAs (111) substrate at $350{ }^{\circ} \mathrm{C}$, mainly focusing on Ge nanowires parallel to the GaAs (111)B surface. The arrows indicate those nanowires. The scale bar is $1 \mu \mathrm{m}$.

view FESEM image will be $67.7^{\circ}$. After careful inspection, we found no such Ge nanowires with an inclined angle of $67.7^{\circ}$ relative to the surface normal. Instead, we observed may kinked Ge nanowires, which had grown parallel to the (111) surface, as shown in Figure 4(c). Similarly, we observed many kinked Ge nanowires on GaAs (100). These kinked nanowires form a group of nanowires showing rectangular symmetry with directions parallel to cleavage direction, as described above. Therefore, the growth direction of these kinked nanowires is one of [0-11], [01-1], [1-10], and [-101], which are perpendicular to the surface normal regardless of the substrate's orientation. All these nanowires can, therefore, be included in the group of the $<110>$ nanowires.

A question may arise as to why the epitaxial Ge nanowires have a strong tendency to grow along the $<110>$ direction when they grow on a GaAs substrate. At present, we cannot draw any decisive conclusion. However, we can propose a plausible scenario. At the initial stage of growth, a certain amount of As/Ga may dissolve in the $\mathrm{Au}$ nanoparticles. In this case, As/Ga will be incorporated into Ge nanowire, and this effect will change the lattice parameter of Ge nanowires during the initial growth. For induced strain to be minimized, Ge nanowires may choose $<110>$ rather than $<111>$ as their growth direction. To prove this hypothesis, a detailed transmission electron microscopic study on the atomic arrangement of Ge should be conducted. In addition, exploring the As/Ga content in an individual Ge nanowire along growth direction should be conducted by 
using detailed energy dispersive X-ray analysis. This will be the subject of our further study.

\section{CONCLUSION}

To conclude, we successfully synthesized epitaxial Ge nanowires on GaAs (100) and GaAs (111)B substrates. In contrast to previously reported epitaxial Ge nanowires on $\mathrm{Si}$ and Ge substrates, which grow predominantly along the $<111>$ direction, the growth direction of Ge nanowires on GaAs is the $<110>$ direction, irrespective of the substrate orientation. In addition, tapering, which is an undesirable property for practical applications, is not substantial at $350{ }^{\circ} \mathrm{C}$. This is in sharp contrast with epitaxial Ge nanowires grown on Ge and Si substrates, which are highly tapered at this temperature. Therefore, epitaxial Ge nanowires grown on GaAs may be important for future nanowire-based optoelectronic devices, including mid-infrared detectors and waveguides.

\section{ACKNOWLEDGMENTS}

This work was supported by a Korea Research Foundation grant (KRF-2006-013-C00106).

\section{REFERENCES}

[1] S. K. Ghandhi, VLSI Fabrication Principles (Wiley, New York, 1994).

[2] S. M. Sze, Physics of Semiconductor Devices (Wiley, New York, 1981).

[3] B. Salazar-Hernandez, M. A. Vidal, H. NavarroContreras and C. Vazquez-Lopez, Thin Solid Films 352, 269 (1999) and references therein.

[4] M. Dubey, K. A. Jones, D. W. Eckart, L. M. Casas and R. L. Pfeffer, Appl. Phys. Lett. 64, 2697 (1994).

[5] R. S. Wagner and W. C. Ellis, Appl. Phys. Lett. 4, 89 (1964).

[6] C. M. Lieber, MRS Bull. 28, 486 (2003).
[7] X. Duan, Y. Huang, R. Agawal and C. M. Lieber, Nature 421, 241 (2003).

[8] M. H. Huang, S. Mao, H. Feick, H. Yan, Y. Wu, H. Kind, E. Weber, R. Russo and P. Yang, Science 292, 1897 (2001).

[9] M. T. Bjork, B. J. Ohlsson, C. Thelander, A. I. Persson, K. Deppert, L. R. Wallenberg and L. Samuelson, Appl. Phys. Lett. 81, 4458 (2002).

[10] C. Thelander, H. A. Nilsson, L. E. Jensen and L. Samuelson, Nano Lett. 4, 1987 (2005).

[11] O. Hayden, R. Agarwal and C. M. Lieber, Nat. Mater. 5, 352 (2006)

[12] L. J. Lauhon, M. S. Gudiksen and C. M. Lieber, Phil. Trans. R. Soc. London A 362, 1247 (2004).

[13] Y. S. Park, J. C. Lee and T. W. Kang, J. Korean Phys. Soc. 49, 2010 (2006).

[14] J. Y. Kim, H. W. Shim, E. K. Suh, T. Y. Kim, S. H. Lee, Y. H. Mo and K. S. Nahm, J. Korean Phys. Soc. 44, 137 (2004).

[15] H. W. Kim, S. H. Shim and C. Lee, J. Korean Phys. Soc. 49, 628 (2006).

[16] J. S. Kim, W. I. Park, C.-H. Lee and G.-C. Yi, J. Korean Phys. Soc. 49, 1635 (2006).

[17] Y. Kim, Q. Gao, H. J. Joyce, H. H. Tan, C. Jagadish, M. Paladugu and J. Zou, Proc. of SPIE 6352, 635226 (2006) and references therein.

[18] D. Wang and H. Dai, Angew. Chem. 114, 4977 (2002).

[19] T. I. Kamins, X. Li, R. S. Williams and X. Liu, Nano Lett. 4, 503 (2004).

[20] P. Nguyen, H. T. Ng and M. Meyyappan, Adv. Mater. 17, 549 (2005).

[21] J. W. Dailey, J. Taraci, T. Clement, D. J. Smith, J. Drucker and S. T. Picraux, J. Appl. Phys. 96, 7556 (2004).

[22] H. Adhikari, A. F. Marshall, C. E. D. Chidsey and P. C. McIntyre, Nano Lett. 6, 318 (2006).

[23] H. Jagannathan, M. Deal, Y. Nishi, J. Woodruff, C. Chidsey and P. C. McIntyre, J. Appl. Phys. 100, 024318 (2006).

[24] E. Tutuc, S. Guah and J. O. Chu, Appl. Phys. Lett. 88, 043113 (2006).

[25] Y. Kim, H. J. Joyce, Q. Gao, H. H. Tan, C. Jagadish, M. Paladugu, J. Zou and A. A. Suvorova, Nano Lett. 6, 599 (2006).

[26] A. B. Greytak, L. J. Lauhon, M. S. Gudikson and C. M. Lieber, Appl. Phys. Lett. 84, 4176 (2004). 